\title{
Transients in the load node at power loss: group run-out of induction motors
}

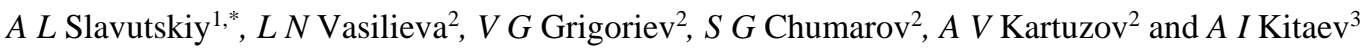 \\ ${ }^{1}$ Software products development, Separate division of LLC "Unitel Engineering" in Cheboksary, 2-nd Kabelnaya str., 2, building 1, \\ 111024 Moscow, Russia \\ ${ }^{2}$ Faculty of Radio Electronics and Automatics, Chuvash State University, Moskovskiy pr.,15, Cheboksary, 428015, Russia \\ ${ }^{3}$ Department of Mathematics and Physics, Chuvash State Pedagogical University, K. Marx st., 38, Cheboksary, 428000, Russia
}

\begin{abstract}
The simulation of transient processes in the complex load node with powerful induction motors at the moment of power loss is carried out. For the modeling the method of synthetic schemes (Dommel's algorithm) was used. Calculations are carried out within the dynamic model of motors in phase coordinates. The results of simulation and analysis modes of the load node with two induction motors connected to the electric buses of $10 \mathrm{kV}$ and fed through a step-down transformer with $16 \mathrm{MVA}$ capacity are presented. The applied model of power transformer consists of inductively coupled branches. The features of single and joint run-out of motors with different torque of mechanical loads are analyzed. Estimates of the parameters and time intervals at which the run-out of the motors is close to synchronous are obtained, the features of energy recuperation and the interaction of the motors in the load node are analyzed.
\end{abstract}

\section{Introduction}

For the analysis of transients in the complex load nodes it is often necessary to study the operating modes of the node with powerful induction motors [1-3]. One of the important modes for the analysis is the run-out of the induction motor with energy recovery in the electric network. The presence of several motors in the load node imposes features on the run-out mode, since the joint run-out of several motors is accompanied by an exchange of energy between the motors and the disconnected section of the power system.

From a practical point of view, self-starting of electric motors is an important topic for research $[4,5]$. Self-starting usually follows a run-out of the motor, and the topic of the run-out is regarded as the initial conditions for the analysis of self-starting after a power loss, as it is, very often, in the uncontrollable mode. When self-starting, conditions arise when the motor or a group of motors fall into a state in which they can not start without changing the parameters of the electric network or mechanical load. This fact makes the simulation of the motors group relevant from a practical point of view.

\section{Methods}

Calculation of transients was made using the method of synthetic schemes (Dommel's Algorithm) [6]. The peculiarity of this method is that the simulation results are obtained in the form of instantaneous values of quantities with a given discretization in time. At the same time, the solved system of equations at each step completely describes the topology of the studied scheme, which makes it easy to link the models of various nonlinear elements.

There are different methods of calculation of the transient processes in an induction machine. The most common method of calculating the modes of the motor in the electric circuit is to account for it in the form of a replacement circuit [7]. In this case, the active resistance of the rotor circuit depends on the sliding of the motor. This approach is the easiest when taking into account the motor in the electric scheme and is a compact mathematical model of the machine. However, the simplicity of such a model is associated with many assumptions and leads to low accuracy of the results, especially for the simulation of nonstationary modes.

This model is not suitable for use in conjunction with the method of synthetic circuits, since it involves the use of the effective values in the calculation of mechanical parameters of the machine - torque, angular velocity, etc. Dynamic models of induction motors [8,9], as a rule, have a more complex structure and are presented in coordinates associated with the rotor or stator of the machine, for example, coordinates $(d, q, 0),(\alpha, \beta, 0)$, etc. [10-12].

In this paper, a model in phase coordinates is applied. This choice is due to the simplicity of matching the electric network model and the motor model - the network and the motor are modeled as three-phase systems, which allows us to directly connect the clamps of the motor model to the three-phase network model without additional transformations of the coordinate

\footnotetext{
* Corresponding author: las co@mail.ru
} 
systems [10]. The system of phase coordinates is convenient for its visibility and makes it easy to simulate complex symmetric and asymmetric modes of multiphase electric network.

The power transformer model consisting of inductively coupled branches is used. This model allows to perform the calculation taking into account the transformation of voltage levels [2] and groups of connection of transformer windings. The parameters of the model are calculated from the catalog data of transformers. The models of three-phase transformers in the form of a system of three single-phase transformers are used. This approach is applicable to both twowinding and three-winding transformers. At the same time, depending on the connection scheme of singlephase transformers, it is possible to obtain different groups of connection of windings of three-phase transformers.

\section{Results and discussion}

Let us analyze the load node modes with two powerful induction motors. The scheme (Figure 1) with two induction motors connected to a $10 \mathrm{kV}$ bus and fed through a transformer is considered. The source on the $110 \mathrm{kV}$ side is replaced by an equivalent voltage source with internal resistance. The simulation is carried out to load node with two induction motors (the"ASMP-5000" is the nominal power of $5000 \mathrm{~kW}$ ). The motors are fed from a transformer with a power of $16 \mathrm{MVA}$.

Thus, based on the simulation results, it is possible to assess the mutual influence of two induction motors operating in one complex load node during their run-out. This influence extends both to electromagnetic transients - change of currents in windings of motors, and electromechanical - changes of the moments and angular speeds of both motors at change of modes of one of them.

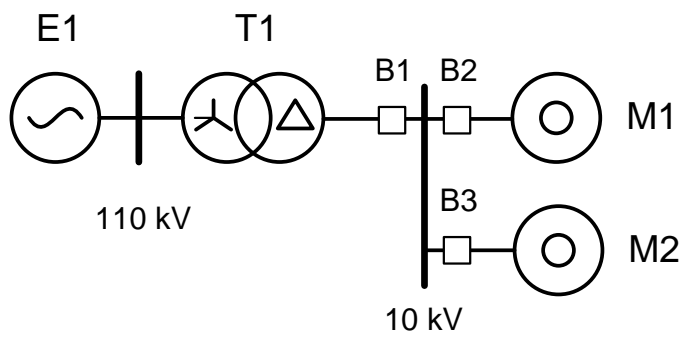

Fig. 1. Scheme of the load node investigated.

We will consider the mode of joint run-out of these motors. For clarity, the moments on the motor shaft are assumed different. The M1 motor has a resistance torque on the shaft of $500 \mathrm{~N}-\mathrm{m}$, for the M2 motor the fan load $[5,13]$ is given in the expression:

At the time the simulation starts $(0 \mathrm{~s})$ all switches are on and the motors are operating in a stationary mode. At the time of $0.1 \mathrm{~s}$ the switch B1 is switched off and the motors remain connected to one bus without external power and additional electrical load. The motors begin to overrun the joint, working to a common bus. To simplify, figures 2 and 3 show the currents and voltages of only one stator phase of each motor, since the motors operate in a symmetrical mode and there are no transients that violate the symmetry of the currents and phase voltages.
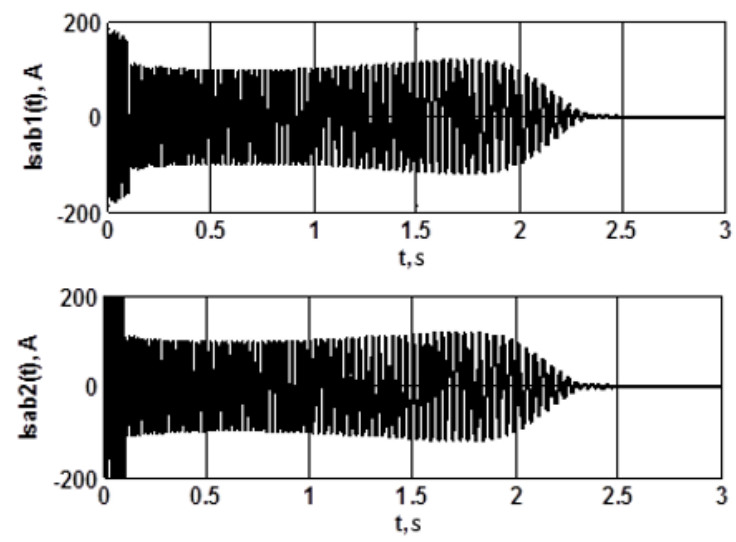

Fig. 2. Graphs of phase currents of stators of the motors in a joint run-out. Isab1 - for the motor M1, Isab2 - for the motor M2.

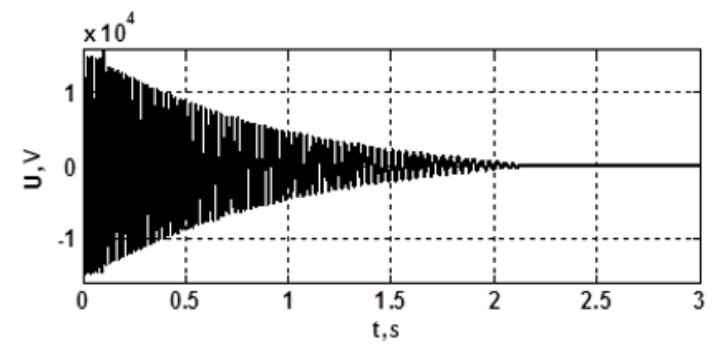

Fig. 3. Graph of voltage on windings A-B of the stators of the motors in a joint run-out.

Consider the graphs of the angular velocity of both motors in figure 4 . At the beginning of the run-out process angular velocities of both motors are very close. We can say that in the first second the change in the speed of rotation of the motors is almost synchronous [1].

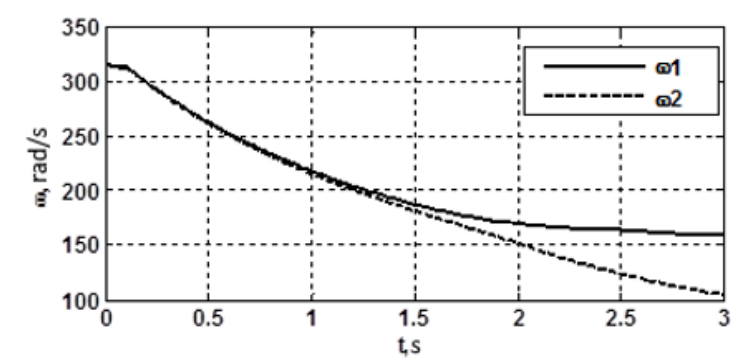

Fig. 4. Graphs of angular velocities of motors at joint run-out. $\omega 1$ - for the motor M1, $\omega 2$ - for the motor M2.

The graphs in figure 5 show that during the joint runout, the electromagnetic moments of the motors become equal in modulus and opposite in sign. This fact suggests that one motor during the run-out works in the generator mode, and the second - in the motor mode. The moment $\mathrm{T} 1$ of the motor M1 is negative - it works in the generator mode, and the moment $\mathrm{T} 2$ of the motor M2 is 
positive - it works in the motor mode. Recall that the mechanical moment of resistance of the motor M1 is fixed, and is $500 \mathrm{~N}-\mathrm{m}$, and the motor M2 is given a fan type load, which at a nominal angular velocity gives the moment of resistance $14800 \mathrm{~N}-\mathrm{m}$.

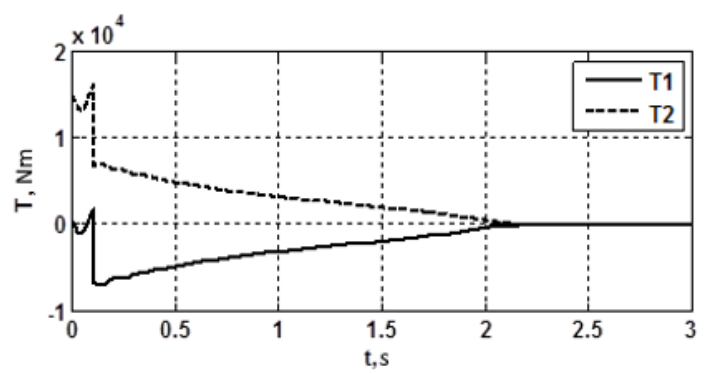

Fig. 5. Graphics of electromagnetic moments of motors at joint run-out. T1- for motor M1, T2 - for motor M2.

For comparison, consider a single run-out mode of both motors with the same load and other parameters of the model. Graphs of the angular velocities of both motors for this mode are shown in Figure 6.

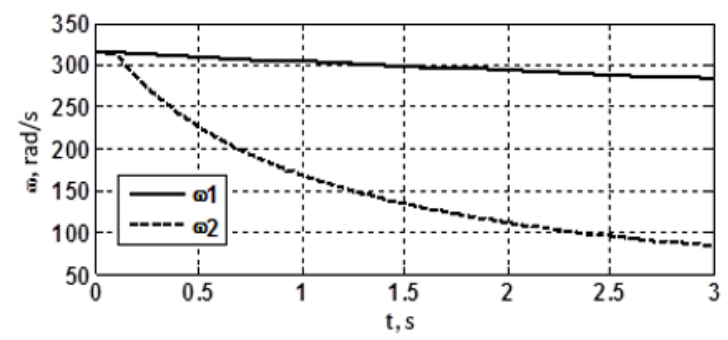

Fig. 6. Graphs of angular speeds of the motors with a single run-out. $\omega 1$ - for the motor M1, $\omega 2$ - for the motor M2.

Comparing angular velocities of motors at joint and single run-out, we will notice that at single run-out the motor M1 with loading of $500 \mathrm{~N}-\mathrm{m}$ (smaller) stops much more slowly, than at joint run-out. So, for example at joint run-out speed of $300 \mathrm{rad} / \mathrm{s}$ is reached by it for $0.1 \mathrm{~s}$. after power failure, and at single run-out - for $1.2 \mathrm{~s}$. The motor M2 with fan type load (bigger) runs out a little more slowly. So, at joint run-out, it reaches the speed of $150 \mathrm{rad} / \mathrm{s}$ at $1.9 \mathrm{~s}$ after power failure, and at single runout - at $1.2 \mathrm{~s}$. Thus, the joint run-out leads to a faster stop of the less loaded motor and a slower stop of the more loaded motor.

Thus, a characteristic feature of the group run-out mode of induction motors is that the run-out is close to synchronous until the voltage on the power buses decreases to a value of $0.4-0.25$ of the nominal. Comparing the graphs of the voltage on the motor windings and the graphs of the motor sliding during the group run-out, you can see that the sliding of the motors begins to vary approximately one second after the start of the run-out. The voltage on the windings at this point reaches about $4 / 1.41=2.8 \mathrm{kV}$ of the current value, which is $28 \%$ of the nominal voltage.

The analysis shows that, with everything else unchanged, the joint run-out of the two motors is more close to synchronous at a significant time interval after the power is out, which is confirmed by practical and theoretical provisions [1]. This is due to the exchange of power between the motor with a large energy reserve and the motor with a smaller energy reserve by means of equalizing currents in the stator windings.

\section{Acknowledgments}

The authors express their gratitude to professor L. A. Slavutskii for useful discussions.

\section{References}

1. S.I. Gamazin, V.A. Stavtsev, S.A. Tsyruk, Transient processes in power supplying systems with electrical driving load, 421 (1997)

2. A.L. Slavutskiy, V.S. Pryanikov, L.A. Slavutskii, Simulating the transients in a load node with a triple-wound transformer at different voltage levels, Russian Electrical Engineering, 88(7), 412-415 (2017)

3. K.M. Vasyliv, A mathematical model of thermal power plants smoke exhausters induction motors system operation modes, Electrical engineering \& electromechanics. , 3, 19-26 (2017)

4. N. Watson, J. Arillaga, Power System Electromagnetic Transients Simulation, 421 (2007)

5. A.G. Kalinin, A.K. Arakelyan, Analysis of voltage dips during start-ups of electric motors with fan load, Elektrichestvo, 6, 46-50 (2011)

6. H.W. Dommel, Digital Computer Solution of Electromagnetic Transientsin Single- and Multiphase Networks, IEEE Transactionson Power Apparatus and Systems, 88(4), 388-399 (1969)

7. V.V. Moskalenko, Electrical driving Moscow, Academia, 368 (2007)

8. L. Wang, J. Jatskevich, V. Dinavahi, Method of Interfacing Rotating Machine Models in Transient Simulation Programs, IEEE TRASACTIONS ON POWER DELIVERY, 25(2), 891-903 (2010)

9. L. Wang, J. Jatskevich, C. Wang, P. Li, VoltageBehind-Reactance Induction Machine Model for the EMTP-Type Solution, IEEE TRANSCTIONS ON POWER SYSTEMS, 23(3), 1226-1238 (2008)

10. A.L. Slavutskiy, Modeling transients in the load nodes containing the induction motors in phase coordinates, Electrical and data processing facilities and systems, 1, 38-45 (2015)

11. A.B. Vinogradov, Vector control of alternating current electrical driving, 298 (2008)

12. P.C. Krause, O. Wasynczuk, S.D. Sudhoff, Analysis of the machinery and drive systems, 630 (2002)

13. O.N. Yadarova, G.P. Okhotkin, L.A. Slavutskii, A control system of a fan drive based on airflow Doppler ultrasonic control, Russian Electrical Engineering, 7, 420-422 (2017) 